\title{
Pattern Synthesis With a Flush-Mounted Leaky- Wave Antenna on a Conducting Circular Cylinder ${ }^{1}$
}

\author{
Akira Ishimaru and Frederick R. Beich \\ Contribution From Department of Electrical Engineering, College of Engineering, \\ University of Washington, Seattle, Wash.
}

(Received May 17, 1962)

\begin{abstract}
This paper is concerned with the synthesis of a radiation pattern in an equatorial plane surrounding an infinite circular cylinder. A discussion of the radiation field about such a cylinder leads to the conclusion that a leaky-wave antenna can be utilized to provide the proper aperture distribution. With $k a$ chosen sufficiently large to ensure that prior theory concerning the inductive sheet leaky-wave antenna will apply, a Chebyshev distribution is analyzed. It is shown that the order of the Chebyshev polynominal must satisfy $N<k a$ $\cos \phi_{0} \sin \delta_{N}$, where $\phi_{0}$ is the angle of radiation measured from a normal to the cylinder surface, and $\delta_{N}$ is the maximum deviation from this angle. The radiation angle is given by $\phi_{0}=\operatorname{arc}$ $\sin (\beta / k)$, where $\beta$ is a constant which is the phase factor of an $X$-band waveguide. Experimental work with such a waveguide, curved in the $H$ plane and flush-mounted in a finite cylinder with $k a=12 \pi$, provided radiation patterns which closely adhered to the theoretical analysis in the frequency range 8.4 to $11.5 \mathrm{Gc} / \mathrm{s}$.
\end{abstract}

\section{Introduction}

The flush-mounted leaky-wave antenna has been the subject of many investigations and resulting papers. Goldstone and Oliner [1959] have contributed to the theory by working with the general leaky-wave structure and applying a perturbation technique to the transverse resonance condition. On the other hand, Honey [1959] has used a computer to solve the transverse resonance equation for the specific case of the inductive sheet antenna. The extension of this type of antenna to the case of a curved surface, namely an infinite circular cylinder, is the purpose of this report.

The far field patterns of a source on an infinite, conducting, circular cylinder have long been known [Silver and Saunders, 1950]. More recently, Wait [1959] has published an entire book on the subject and related topics which also contains a comprehensive bibliography. Wait and Householder [1959] have also obtained theoretical patterns for the slotted cylinder antenna. However, in order to obtain their patterns, it is necessary to control the phase shift around the cylinder in such a manner that rather complicated excitation devices would be needed. The leaky-wave antenna provides a means to avoid such devices.

In this report, theory is developed to show that the radiation field in the equatorial plane surrounding the circular cylinder, except for an azimuthal phase shift, is essentially directed in proportion to the vertically polarized aperture distribution of the leaky-wave antenna.

A Chebyshev distribution is analyzed so that synthesis can be carried out. Subsequent experimental work with the resulting antenna provided justification for the theory, as excellent correspondence between the theoretical radiation pattern and actual radiation patterns over the range 8.4 to $11.5 \mathrm{Gc} / \mathrm{s}$ was demonstrated.

${ }^{1}$ The research reported in this document has been sponsored by the Electronics Research Directorate of the Air Force Cambridge Research Laboratories, Office of Aerospace Research, United States Air Force, Bedford, Mass., under Contract No. AF 19(604)-4098. 


\section{Radiation Field}

Consider excitation of a transverse slot in an infinite conducting cylinder shown in figure 1a as given by

$$
f(\phi) e^{-j \beta a_{\phi}}=\sum_{m=-N}^{N} A_{m} e^{-j m_{\phi}} e^{-j \beta a_{\phi}},-l<z<l,
$$

where $f(\phi)$ is the amplitude distribution in the slot, $\beta$ is the slot phase factor, $a$ is the cylinder radius, $A_{m}$ is the Fourier coefficient, $N$ is an integer, and $2 l$ is the slot width. Following an analysis similar to that of Silver and Saunders [1950], the tangential field is

$$
\begin{gathered}
E_{z}(a, \phi, z,)=\frac{1}{2 \pi} \sum_{m=-N}^{N} e^{-j(m+\beta a) \phi} \int_{\infty}^{\infty}\left(\frac{\sin h l}{h l}\right) A_{m} 2 l e^{-j h z} d h, \\
E_{\phi}(a, \phi, z)=0 .
\end{gathered}
$$

When expanding the field external to the cylinder, the condition of periodicity in the $\phi$-direction is no longer stipulated. It is assumed instead that a traveling wave continuously circles the cylinder. It is further assumed that after one circumvention of the cylinder, the amplitude of the wave becomes negligible such that transmitted or reflected components are of little importance. The $\phi$-dependence under such assumptions is given by

$$
A(\phi) e^{-j \bar{\beta} \phi}
$$

where $\bar{\beta}$ is the phase factor of the external field and $A(\phi)$ is the amplitude function.

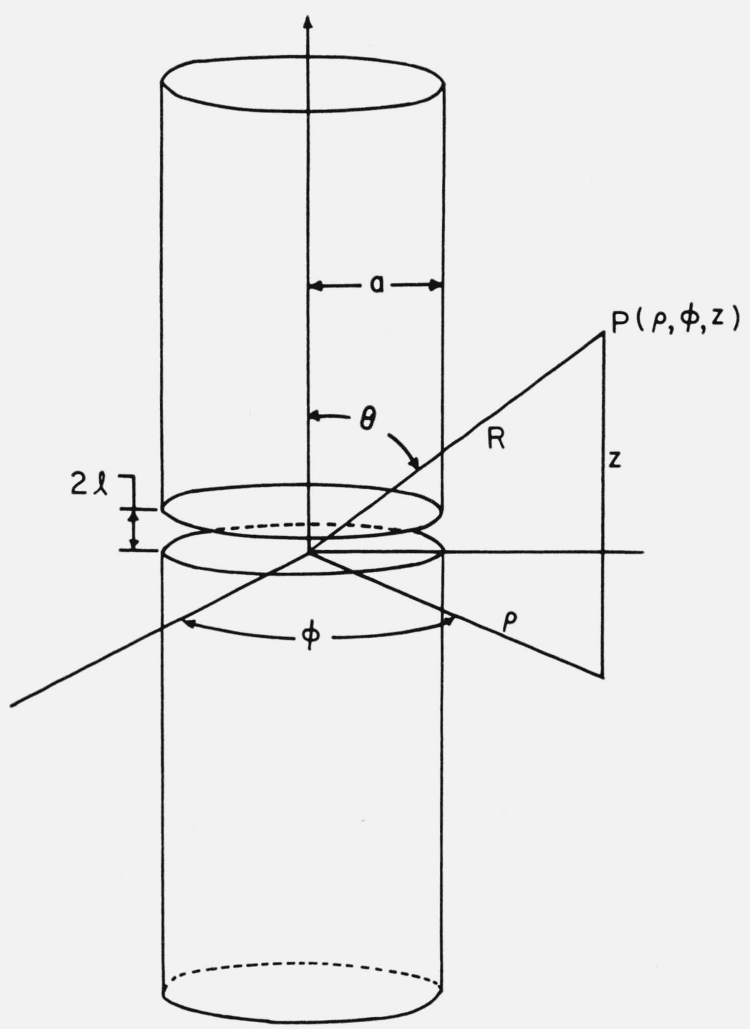

Figure 1a. A transverse slot on an infinite conducting cylinder. 
Although $A(\phi)$ is not periodic on the interval 0 to $2 \pi$, a Fourier series representation over this interval of the form

$$
A(\phi)=\sum_{m=-\infty}^{\infty} c_{m} e^{-j m_{\phi}}
$$

is assumed. Then, by an extension of the results of previous workers [Silver and Saunders, 1950; Wait, 1959], the external field components are found to be

$$
\begin{aligned}
E_{z}(\rho, \phi, z)=\int_{-\infty}^{\infty} \sum_{m=-N}^{N} a_{m} k_{c}^{2} H_{m+\bar{\beta}}^{2}\left(k_{c} \rho\right) e^{-j(m+\bar{\beta})} e^{-j h z} d h, \\
E_{\phi}(\rho, \phi, z)=\int_{-\infty}^{\infty} \sum_{m=-N}^{N}\left[\frac{-(m+\bar{\beta}) h}{\rho} a_{m} H_{m+\bar{\beta}}^{(2)}\left(k_{c} \rho\right)+j \omega \mu b_{m} \frac{\partial}{\partial \rho} H_{m+\bar{\beta}}^{(2)}\left(k_{c} \rho\right)\right] \\
\times e^{-j(m+\bar{\beta}) \phi} e^{-j h z} d h,
\end{aligned}
$$

where $a_{m}$ and $b_{m}$ are arbitrary constants and $k_{c}^{2}=k^{2}-h^{2}, k=\sqrt{\mu_{0} / \epsilon_{0}}$ is the free space propagation constant, and $h$ is the complex propagation factor arising from a Fourier transform in the axial direction. $H_{m+\bar{\beta}}^{(2)}$ is the Hankel function of the second kind and order $m+\bar{\beta}$. Evaluating the latter equations at the surface of the cylinder, i.e., $\rho=a$, and then equating them to the tangential field as given by (2), it is apparent that to be equal for all $\phi$,

$$
\bar{\beta}=\beta a
$$

which then allows $a_{m}$ and $b_{m}$ to be determined. With these constants evaluated, all remaining field components can be found. Next, letting

$$
\rho=R \sin \theta, z=R \cos \theta,
$$

it is apparent that in the radiation field where $R \rightarrow \infty$,

$$
\frac{m+\beta a}{k_{c} R \sin \theta} \rightarrow 0 \text {. }
$$

This means that the usual asymptotic form of the Hankel function can be utilized, and a saddle-point integration will give as the radiation field components,

$$
\begin{gathered}
E_{\theta}=\left(\frac{\sin (k l \cos \theta)}{k l \cos \theta}\right) \sum_{m=-N}^{N} e^{-j \nu_{m} \phi} \frac{\left(-A_{m} 2 l\right) e^{j\left(\nu_{m}+1\right) \pi / 2}}{\pi \sin \theta H_{\nu_{m}}^{(2)}(k a \sin \theta)} \frac{e^{-j k R}}{R} ; H_{\phi}=\frac{E_{\theta}}{\eta}, \\
E_{\phi}=\left(\frac{\sin (k l \cos \theta)}{k l \cos \theta}\right) \sum_{m=-N}^{N} e^{-j \nu_{m} \phi} \frac{A_{m} 2 l\left(-j \nu_{m} \operatorname{ctn} \theta\right) e^{j\left(\nu_{m}+1\right) \pi / 2}}{\pi a \frac{\partial}{\partial a} H_{\nu_{m}}^{(2)}(k a \sin \theta)} \frac{e^{-j k R}}{R} ; H_{\theta}=\frac{-E_{\phi}}{\eta}
\end{gathered}
$$

where $\nu_{m}=m+\beta a, \eta=\sqrt{\mu_{0} / \epsilon_{0}}, H_{\nu_{m}}^{(2)}$ is the Hankel function of second kind and order $\nu_{m}$, and terms that vary as $1 / R^{2}$ have been neglected.

If attention be confined to the equatorial plane only, that is, where $\theta=\pi / 2$, then $E_{\phi}=$ $H_{\theta}=0$. The remaining components, $E_{\theta}$ and $H_{\phi}$, although complicated due to the presence of the Hankel function, can be simplified if the relationship between its order and argument be prescribed such that

$$
\nu_{m}<k a
$$

or

$$
\frac{\nu_{m}}{k a}<1
$$

As shown subsequently in paragraph 3, the ratio in (10b), unlike that of (8), does not approach 
zero, and use must be made of the asymptotic form [Sommerfeld, 1949]

$$
H_{\nu_{m}}^{(2)}(k a)=\sqrt{\frac{2}{\pi k a \sin \alpha}} e^{-j k a(\sin \alpha-\alpha \cos \alpha)+j \pi / 4}
$$

where

$$
\cos \alpha=\frac{\nu_{m}}{k a}
$$

Then, substituting this asymptotic form into the expressions for the remaining field components in the equatorial plane,

$$
E_{\theta}=\sum_{m=-N}^{N} B_{m} A_{m} e^{-j_{v_{m}(\phi+\alpha-\pi / 2)}} e^{-j k(R-a \sin \alpha)} e^{j \pi / 4}
$$

where $B_{m}=-(l / R)(2 k a \sin \alpha / \pi)^{1 / 2}$, and the $H$ component has been dropped since the radiation field is plane polarized.

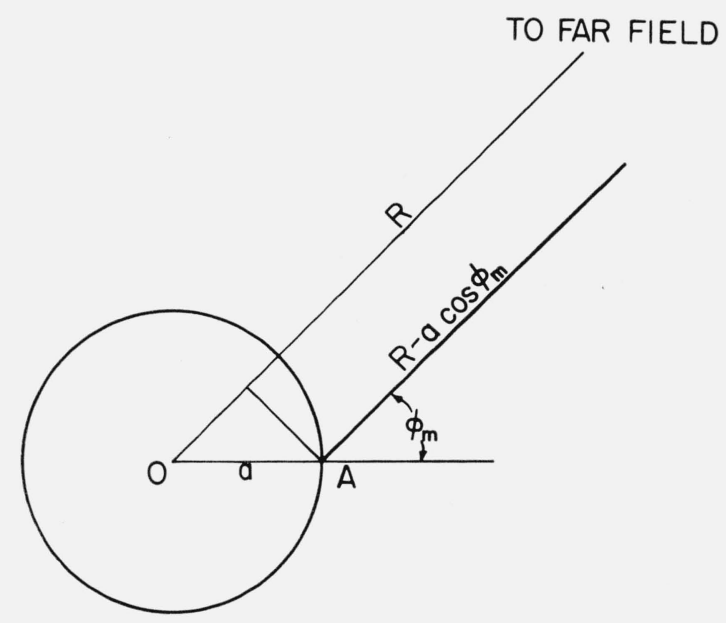

Figure 1b. A plane wave leaving the cylinder in the equatorial plane.

In figure $1 \mathrm{~b}$ is shown the equatorial plane. If a plane wave leaves point $A$ on the surface of the cylinder so as to make an angle $\phi_{m}$ with the normal, and if the space phase difference is measured with respect to the center of the cylinder 0 , then the far field is given by

$$
e^{-j k\left(R-a \cos \phi_{m}\right)},
$$

where $k$ is the free space propagation constant, and the remaining parameters are defined in the figure. Comparing this expression with the radiation field representation of (13), it is apparent that

$$
\cos \phi_{m}=\sin \alpha,
$$

and

$$
\alpha=\pi / 2-\phi_{m} .
$$

Furthermore, from (12) and (16), the radiation angle is defined by

or

$$
\phi_{m}=\arcsin \left(\nu_{m} / k a\right) \text {, }
$$

$$
\phi_{m}=\arcsin \left[\frac{\beta}{k}+\frac{m}{k a}\right]
$$


Therefore (13) can be written

$$
E_{\theta}=\sum_{m=-N}^{N} B_{m} A_{m} e^{-j_{\nu_{m}}\left(\phi-\phi_{m}\right)} e^{-j k\left(R-a \cos \phi_{m}\right)} e^{j \pi / 4} .
$$

Now, let the radiation angle be written

where

$$
\phi_{m}=\phi_{0}+\delta_{m},
$$

$$
\phi_{0}=\arcsin (\beta / k) \text {, }
$$

and $\delta_{m}(m \neq 0)$ is a deviation angle which is a function of the summation index $m$. If the deviation angle is small, then

and (19) reduces to

$$
\cos \phi_{m} \simeq \cos \phi_{0}-\frac{\beta}{k} \delta_{m}
$$

$$
E_{\theta}=B \sum_{m=-N}^{N} \epsilon_{m} A_{m} e^{-j m\left(\phi-\phi_{0}-\delta_{m}\right)} e^{-j \beta a\left(\phi-\phi_{0}\right)}
$$

where

$$
\begin{gathered}
B=-\frac{l}{R} \sqrt{\frac{2 a\left(k^{2}-\beta^{2}\right)^{\frac{1}{2}}}{\pi}} e^{-j k\left(R-a \cos \phi_{0}\right)+j \pi / 4} \\
\epsilon_{m}=\left[1-\frac{\delta_{m}}{\sqrt{\left(k^{2} / \beta^{2}\right)-1}}\right]
\end{gathered}
$$

Under the restriction that $\delta_{m}$ be small, the radiation field of (23), except for a phase shift of $\phi_{0}$ degrees, will be directly proportional to the slot distribution of (1). It can be concluded, therefore, that a desired pattern can be synthesized in the equatorial plane simply by controlling the excitation of the aperture.

\section{Aperture Excitation}

It is necessary to determine how the excitation in the aperture given by (1) should be accomplished. Honey [1959] has suggested that the inductive sheet antenna might be used on a curved surface. In the case of the circular cylinder, values of $k a$ sufficiently large should ensure that his analysis can be applied directly.

The inductive sheet used in this case will consist of the outer wall of an $X$-band waveguide curved in the $H$ plane and propagating a $\mathrm{TE}_{10}$ mode. Although an approximate solution exists [Borgnis and Papas, 1958] for the propagation factor of such a guide, it is not in a readily usable form, and the assumption that $k a$ is large allows use of the approximate dependency $e^{-\gamma_{s} s} e^{-\gamma} \tau^{r}$, where $r, s, z$ form the coordinate system of figure 2a. The wave equation is thus

$$
\gamma_{r}^{2}+\gamma_{s}^{2}+k^{2}=0
$$

where the propagation factors $\gamma_{s}$ and $\gamma_{r}$ are for the longitudinal and transverse directions respectively.

Due to the fact that energy is gradually being "leaked" from the waveguide, the longitudinal propagation factor will be complex,

$$
\gamma_{s}=\alpha+j \beta
$$

The imaginary part of this expression is, of course, just the phase factor of (1). Since it does not vary with the azimuthal angle $\phi$,

$$
\beta=\text { constant } .
$$




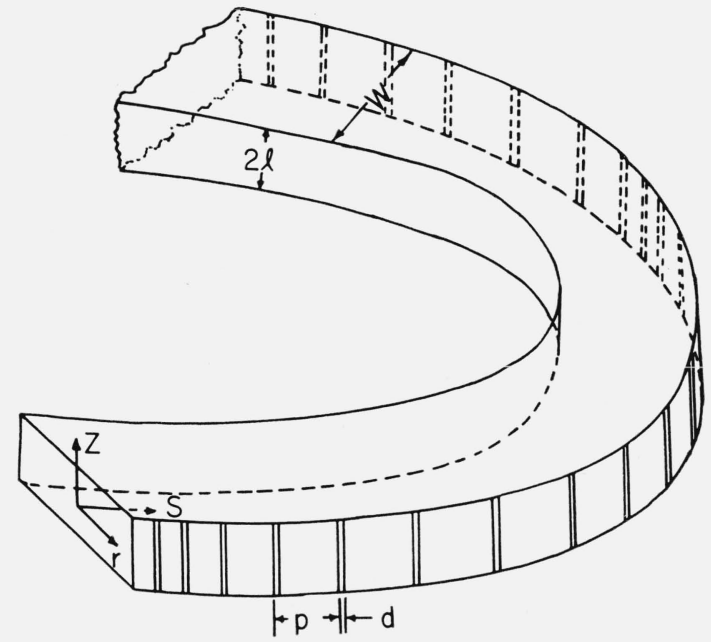

Figure 2a. Inductive wall waveguide and coordinate system.

In addition, because the waveguide is propagating a fast wave, or leaky wave, it is possible to write

$$
0<\frac{\beta}{k}<1
$$

or utilizing (21)

$$
0<\phi_{0}<\pi / 2 .
$$

Furthermore, if the deviation angle is selected such that

$$
0<\phi_{0}+\delta_{m}<\pi / 2,
$$

then, from (20) and (17), it can be concluded that

$$
0<\frac{\nu_{m}}{k a}<1,
$$

which justifies the use of the asymptotic form of (11).

Turning attention to the real part of the longitudinal propagation factor, it is possible to relate it to the amplitude distribution in the slot since [Honey, 1959]

$$
2 \alpha(s)=\frac{g^{2}(s)}{\frac{1}{F} \int_{0}^{L} g^{2}\left(s^{\prime}\right) d s^{\prime}-\int_{0}^{s} g^{2}\left(s^{\prime}\right) d s^{\prime}},
$$

where $g(s)$ is the amplitude distribution, $F$ is the fraction of the input power which is radiated by the antenna, $L$ is the total length of the aperture, and $s^{\prime}$ is the variable of integration along the aperture.

If $g(s)$ be expanded in a Fourier series as

$$
g(s)=\sum_{m=-\infty}^{\infty} c_{m} e^{-j 2 m \pi s / L}=f\left(\frac{2 \pi s}{L}\right)
$$

then for the curved cylindrical surface $s=a^{\prime} \phi, L=2 \pi a^{\prime}$ and $f\left(\frac{2 \pi s}{L}\right)=f(\phi)$. Hence, the expression for the attenuation factor is

$$
2 \alpha(s)=\frac{\frac{1}{a^{\prime}} f^{2}(\phi)}{\frac{1}{F} \int_{0}^{2 \pi} f^{2}\left(\phi^{\prime}\right) d \phi^{\prime}-\int_{0}^{\theta} f^{2}\left(\phi^{\prime}\right) d \phi^{\prime}},
$$




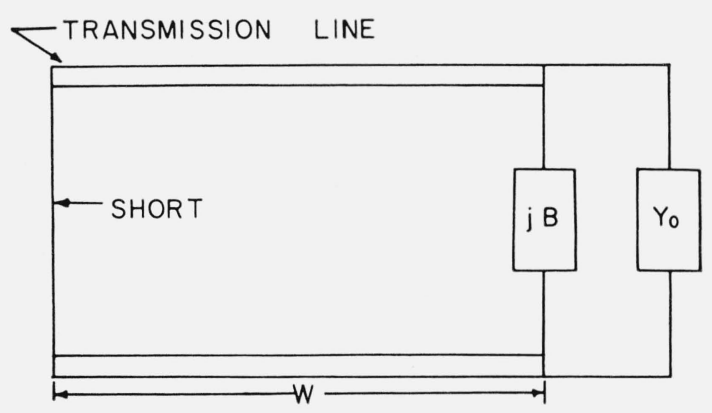

FIGURE 2b. Transverse network represenation.

where $\phi^{\prime}$ is the variable of integration for the $\phi$ dimension, and $a^{\prime}=a-w_{0} / 2$ is the radius which corresponds to the circumferential path in the center of the waveguide, $w_{0}$ being the width of the closed guide.

Having established the longitudinal propagation factor, the next logical step is to find the transverse propagation factor, therefore let

$$
\gamma_{r}=\sigma+j \tau
$$

Substituting this together with (27) into (26) leads to the solution for the real and imaginary parts of $\gamma_{r}$ as

$$
\begin{gathered}
\sigma=-\left[\frac{-\left(\alpha^{2}-\beta^{2}+k^{2}\right)+\sqrt{\left(\alpha^{2}-\beta^{2}+k^{2}\right)^{2}+4(\alpha \beta)^{2}}}{2}\right]^{\frac{1}{2}}, \\
\tau=\left[\frac{\left(\alpha^{2}-\beta^{2}+k^{2}\right)+\sqrt{\left(\alpha^{2}-\beta^{2}+k^{2}\right)^{2}+4(\alpha \beta)^{2}}}{2}\right]^{\frac{1}{2}} .
\end{gathered}
$$

However, another condition to which the inductive sheet antenna must conform is the transverse resonance equation. This condition arises due to the conservation of energy; that is, admittances or impedances must be equal when viewed transversely in either direction from a plane parallel to the inductive sheet wall. Taking as the reference plane the inductive wall itself, the network representation of figure $2 \mathrm{~b}$ is obtained. Therefore, transverse resonance gives rise to the characteristic equation [Honey, 1959]

$$
1+\operatorname{coth} \gamma_{r} w=\frac{-C^{\prime}}{\gamma_{r}}
$$

where $w$ is the width of the waveguide,

$$
C^{\prime}=\frac{2 \pi}{p \ln \csc (\pi d / 2 p)}
$$

and parameters $p$ and $d$ are the strip spacing and width as shown in figure 2a. Writing the characteristic equation in real and imaginary parts,

$$
\begin{gathered}
\tau+\frac{\tau \sinh \sigma w \cosh \sigma w-\sigma \sin \tau w \cos \tau w}{\sinh ^{2} \sigma w \cos ^{2} \tau w+\sin ^{2} \tau w \cosh { }^{2} \sigma w}=0, \\
\sigma+\frac{\sigma \sinh \sigma w \cosh \sigma w+\tau \sin \tau w \cos \tau w}{\sinh ^{2} \sigma w \cos ^{2} \tau w+\sin ^{2} \tau w \cosh ^{2} \sigma w}=-C^{\prime} .
\end{gathered}
$$

At this point it is possible to more clearly define the aperture distribution problem: Find $w$, the waveguide width, and $C^{\prime}$, a parameter of the inductive wall geometry such that

$$
\begin{aligned}
& \alpha\left(w, C^{\prime}\right)=\alpha(s) \\
& \beta\left(w, C^{\prime}\right)=\text { constant. }
\end{aligned}
$$


This means:

(a) $\beta$ should be selected as the propagation factor of the closed waveguide, and thus satisfy (28). (As shown subsequently in par. 4, its selection will also have a bearing on the amplitude distribution.)

(b) Determine the attenuation factor as a function of the circumferential distance from (35).

(c) Find the components of the transverse propagation factor as functions of the circumferential distance from (37).

(d) For each of the components determined in (c), solve the transcendental (40a) for the waveguide width $w$.

(e) For each of the values of $w$ determined, solve (40b) for the inductive wall parameter $C^{\prime}$.

(f) Using each value of $C^{\prime}$ found in (e), find the strip spacing $p$ from (39), or curves [Honey, 1959].

\section{The Chebyshev Distribution}

The pattern whose synthesis is being undertaken is a Chebyshev distribution. As Duhamel [1953] has shown, a Chebysher polynomial can be expanded as a finite Fourier series

$$
T_{N}(x)=T_{N}(a \cos \phi+b)=\sum_{m=0}^{N} C_{m}^{N} \cos m \phi,
$$

where $x=a \cos \phi+b$ and $C_{m}^{N}$ is a function of $a$ and $b$.

However, there are two conditions to which this distribution must adhere. The first condition results from (28), i.e., the phase factor of the distribution must be constant. This in turn implies that the amplitude distribution shall not change sign, thus inherently introducing a phase shift of $\pi$ radians. In the general synthesis with a Chebyshev polynomial this does occur. To combat this difficulty the polynomial $T_{N}(x)+1$ can be utilized [Silver, 1949].

The second condition imposed can be derived from the relationships of (18) and (20) which, when combined, yield

$$
\sin \left(\phi_{0}+\delta_{m}\right)=\frac{\beta}{k}+\frac{m}{k a},
$$

or

$$
m=\beta a\left(\cos \delta_{m}-1\right)+k a \cos \phi_{0} \sin \delta_{m} .
$$

Since $\delta_{m}$ is small and $-N \leq m \leq N$, the restriction becomes

$$
N \leq k a \cos \phi_{0} \sin \delta_{m},
$$

which determines the order of the Chebyshev polynomial to be expanded.

Once the order of the Chebysher polynomial has been determined, it can be used in (35). To illustrate with an example, consider the polynomial

$$
T_{5}(x)+1=16 x^{5}-20 x^{3}+5 x+1=\sum_{m=0}^{5} C_{m}^{5} \cos m \phi,
$$

where [Duhamel, 1953]

$$
\begin{aligned}
& C_{0}^{5}=1+5 b-20 b^{3}+16 b^{5}-30 a^{2} b+80 a^{2} b^{3}+30 a^{4} b^{5} \\
& C_{1}^{5}=5 a-60 a b+8 a b^{4}-15 a^{3}+120 a^{3} b^{2}+10 a^{5} \\
& C_{2}^{5}=-30 a^{2} b+80 a^{2} b^{3}+40 a^{4} b
\end{aligned}
$$


$C_{3}^{5}=-5 a^{3}+40 a^{3} b^{2}+5 a^{5}$

$C_{4}^{5}=10 a^{4} b$

$C_{5}^{5}=a^{5}$.

For this case, the integral in the denominator of (35) is given by

$$
\begin{aligned}
\int_{0}^{\phi} & \left(\sum_{m=0}^{5} C_{m}^{5} \cos m \phi\right)^{2} d \phi=\frac{1}{2}\left(2 C_{0}^{2}+C_{1}^{2}+C_{2}^{2}+C_{3}^{2}+C_{4}^{2}+C_{5}^{2}\right) \phi \\
& +\left(2 C_{0} C_{1}+C_{1} C_{2}+C_{2} C_{3}+C_{3} C_{4}+C_{4} C_{5}\right) \sin \phi \\
& +\frac{1}{4}\left(C_{1}^{2}+4 C_{0} C_{2}+2 C_{1} C_{3}+2 C_{2} C_{4}+2 C_{3} C_{5}\right) \sin 2 \phi \\
& +\frac{1}{3}\left(2 C_{0} C_{3}+C_{1} C_{4}+C_{2} C_{5}+C_{1} C_{2}\right) \sin 3 \phi \\
& +\frac{1}{8}\left(C_{2}^{2}+4 C_{0} C_{4}+2 C_{1} C_{5}+2 C_{1} C_{3}\right) \sin 4 \phi \\
& +\frac{1}{5}\left(2 C_{0} C_{5}+C_{1} C_{4}+C_{2} C_{3}\right) \sin 5 \phi \\
& +\frac{1}{12}\left(C_{3}^{2}+2 C_{2} C_{4}+2 C_{2} C_{4}+2 C_{1} C_{5}\right) \sin 6 \phi \\
& +\frac{1}{7}\left(C_{3} C_{4}+C_{2} C_{5}\right) \sin 7 \phi \\
& +\frac{1}{16}\left(C_{4}^{2}+2 C_{3} C_{5}\right) \sin 8 \phi \\
& +\frac{1}{9}\left(C_{4} C_{5}\right) \sin 9 \phi \\
& +\frac{1}{20}\left(C_{5}^{2}\right) \sin 10 \phi
\end{aligned}
$$

where for convenience the superscript 5 has been dropped from the $C_{m}$ 's. In the general case, similar expressions for any order polynomial may be derived.

\section{Numerical Solution}

A brief description of the numerical solution for the experimental antenna is presented here to illustrate the steps outlined at the end of paragraph 3.

As a preliminary consideration, the operating frequency and cylinder radius must be chosen to ensure that $k a$ is sufficiently large. In this case the design frequency was $10 \mathrm{Gc} / \mathrm{s}$, and a cylinder radius of six wavelengths gave a value of $k a=12 \pi$.

Step (a):

The waveguide propagation factor of standard X-band guide is

$$
\beta=2 \pi / \lambda_{g}=4.01967 \mathrm{rad} / \mathrm{in} .
$$

Also the free space propagation factor of $10 \mathrm{Gc} / \mathrm{s}$ is

$$
k=2 \pi / \lambda=5.3284 \mathrm{rad} / \mathrm{in} .
$$

With these values determined, two additional calculations can be made: 


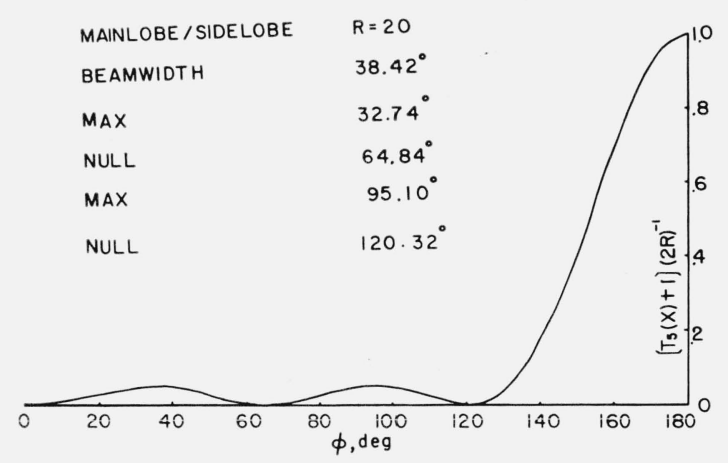

Figure 3. Radiation pattern based on the $T_{5}(x)+1$ polynomial.

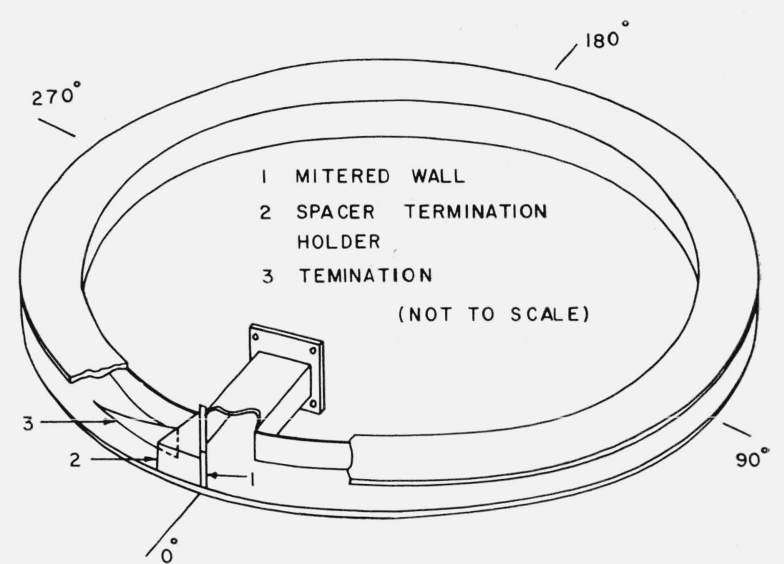

FiguRE 4. Waveguide with grating wall removed to show mitered corner.

First, the unperturbed radiation phase angle from (21) is

$$
\phi_{0}=\arcsin (0.75505)=49.0^{\circ} ;
$$

and second, selecting a maximum deviation angle of 13 degrees (to conform with (31) and the approximation of (22)), the order of the Chebyshev polynomial must satisfy (45),

$$
N \leq(37.8)(0.655)(0.225)=5.58 \text {. }
$$

Letting $N=5$, the distribution as shown in figure 3 can be obtained where $R=20$ is the main lobe to sidelobe ratio corresponding to $26 \mathrm{db}$ down. In figure 5 the theoretical radiation power pattern is shown, where the main lobe appears at $180^{\circ}+49.0^{\circ}=229.0^{\circ}$.

(Note that on the scale shown, the sidelobes will not be visible.)

Steps (b) and (c):

The longitudinal attenuation factor was computed for increments of five degrees (about $\lambda / 2$ on the circumference) using $F=0.9$, and the expression of (48). With the values thus obtained for $\alpha$, calculation of the transverse phase factors was accomplished. Instead of solving for the transverse attenuation factors from (37a), the following relationship was used

Steps (d), (e), and (f):

$$
\sigma=-\alpha \beta / \tau
$$

Computation of the waveguide width $w$ involved the solution of a transcendental equation which must be carried out with the aid of a computer or curves as those given by Honey [1959]. Likewise, the spacing, $p$, of the wires of the inductive wall grating must also be found from a computer solution or from curves.

The results of these calculations are to be found tabulated in appendix A.

\section{Experimental Results}

An infinite cylinder was approximated by fitting two 24 in. (about 20 wavelengths) circular cylindrical sections fabricated from $0.050 \mathrm{in}$. sheet aluminum to the top and bottom of the $X$-band waveguide shown in figure 4 . This waveguide had its inner wall varied so as to maintain the inside width $w$ as computed from (40a). The outer wall consisted of number 28 copper wire fastened to a thin paper backing by overlaying with epoxy resin, and spaced as per dimension $p$ calculated from (39).

Although the design frequency was $10 \mathrm{Gc} / \mathrm{s}$, it was found that the radiation pattern over the range from 8.4 to $11.5 \mathrm{Gc} / \mathrm{s}$ closely approximated the theoretical pattern shown in figure 5 , as can be seen by comparing it with figures 6 to 12 . 


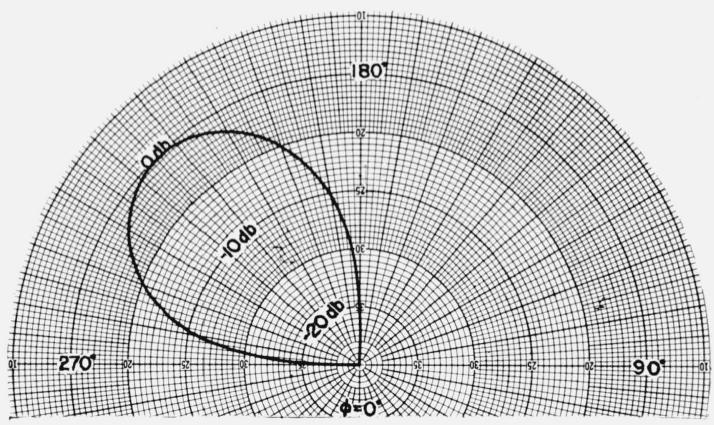

Figure 5. Theorelical pattern for $10 \mathrm{Gc} / \mathrm{s}$.

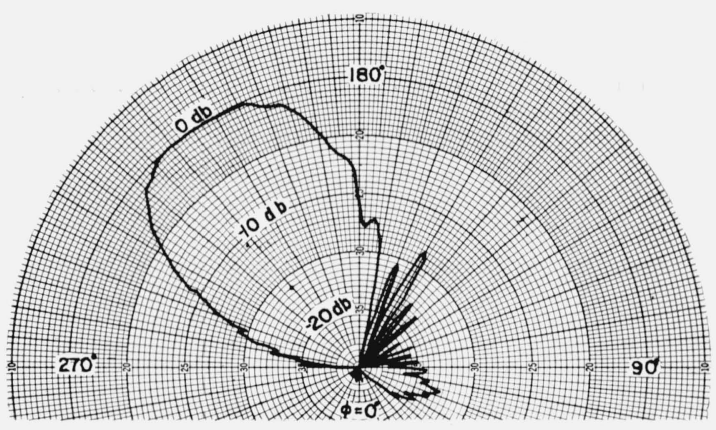

Figure 6. Radiation pattern for $8.4 \mathrm{Gc} / \mathrm{s}$.

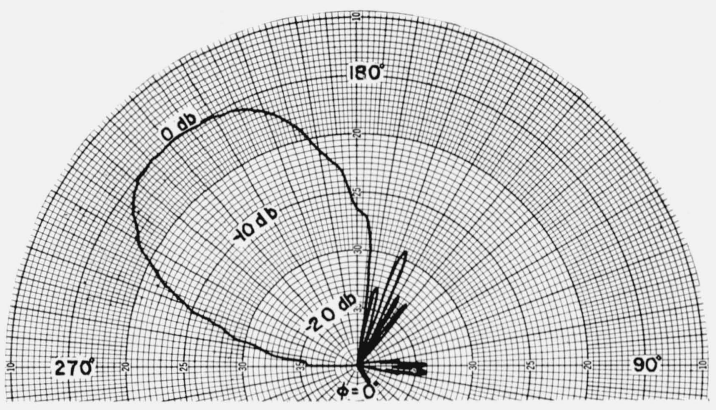

Figure 7. Radiation pattern for $9.0 \mathrm{Gc} / \mathrm{s}$.

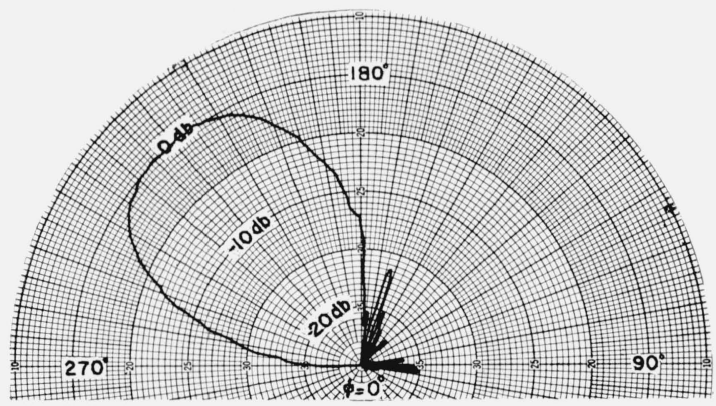

Figure 8. Radiation pattern for $9.5 \mathrm{Gc} / \mathrm{s}$.

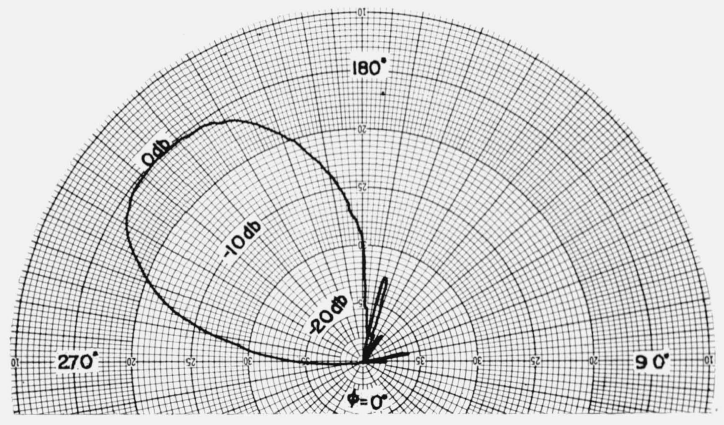

Figure 9. Radiation pattern for $10.0^{-} \mathrm{Gc} / \mathrm{s}$.

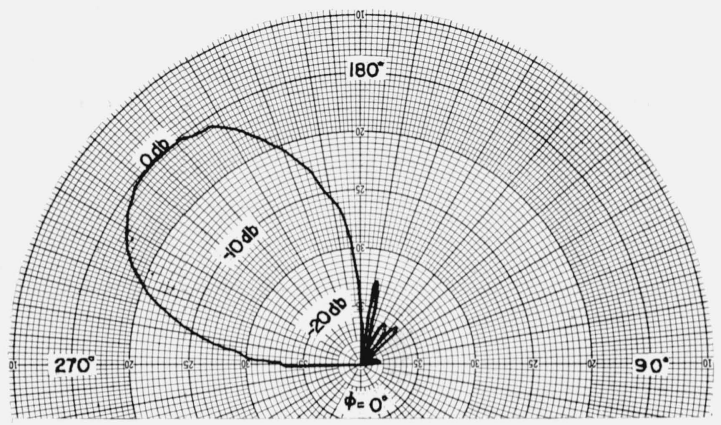

Figure 10. Radiation pattern for $10.5 \mathrm{Gc} / \mathrm{s}$.

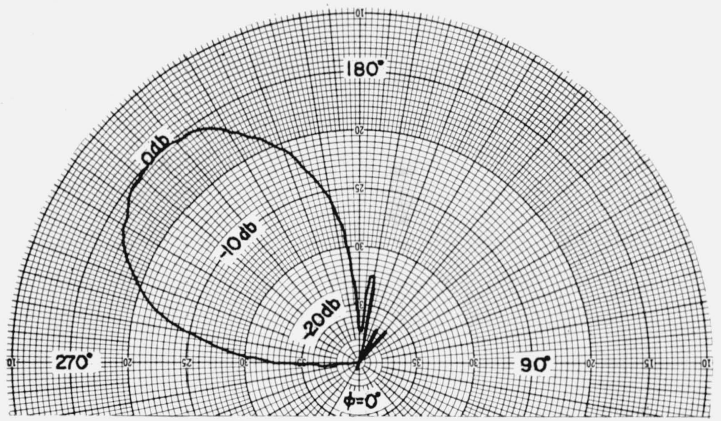

Figure 11. Radiation pattern for $11.0 \mathrm{Gc} / \mathrm{s}$.

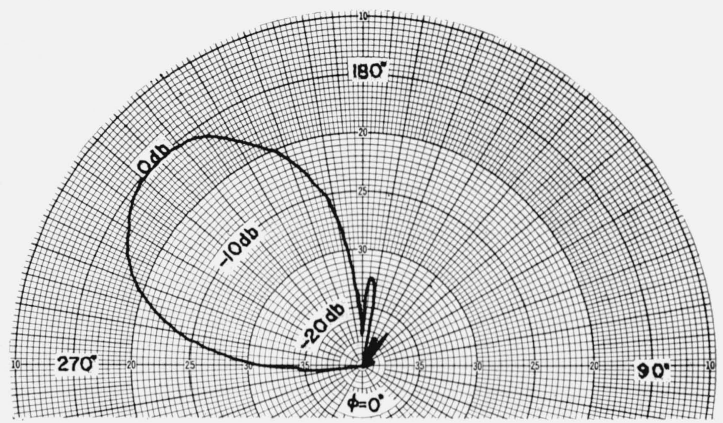

FiguRE 12. Radiation pattern for $11.5 \mathrm{Gc} / \mathrm{s}$. 


\begin{tabular}{|c|c|c|c|c|c|c|c|c|}
\hline & Theory & $8.4^{\mathrm{a}}$ & 9.0 & 9.5 & 10.0 & 10.5 & 11.0 & 11.5 \\
\hline Main beam & $229^{\circ}$ & $215^{\circ}$ & $221^{\circ}$ & $221^{\circ}$ & $224^{\circ}$ & $225^{\circ}$ & $226^{\circ}$ & 22 \\
\hline Sidelobe level (db down). & 26 & 13.5 & 14. 3 & 16. 2 & 17.4 & 17. 6 & 17.3 & 17.3 \\
\hline Beamwidth & $38.4^{\circ}$ & $41^{\circ}$ & $43^{\circ}$ & $42^{\circ}$ & $42^{\circ}$ & $40^{\circ}$ & $38^{\circ}$ & $38^{\circ}$ \\
\hline
\end{tabular}

a Indicates the frequency in $\mathrm{Gc} / \mathrm{s}$ at which radiation pattern was taken.

The main departures from the theoretical pattern are apparent from the data of table 1: The direction of the main beam at the design frequency of $10 \mathrm{Gc} / \mathrm{s}$ is slightly in error; sidelobe levels are higher than theory predicts; and a wider beamwidth exists at the design frequency.

The apparent disagreement in the main beam position is caused, in the main, by the inability to aline the antenna on the pattern range. The circular symmetry of the cylinder allowed no better than a $\pm 3^{\circ}$ tolerance in locating a given point on the circumference, namely the feed point at $0^{\circ}$ (see fig. 4 ).

The disagreement in sidelobe level is due to the spurious sidelobes exhibited in the radiation patterns of figures 6 to 12. These sidelobes could be attributed to several factors: (1) The assumption that the transmitted and reflected components of the azimuthal traveling wave of the external field are negligible may not be valid as certain harmonic components of (4) may cause standing waves to exist; (2) The deviation angle $\delta_{m}$ and $\epsilon_{m}$ defined in (25) show that the radiation field is not, in the strict sense, proportional to the aperture distribution and the effect of these perturbations may cause the sidelobes; (3) A correction term in (39) which was neglected [Honey, 1959] could also account for premature excess leakage of energy giving rise to sidelobes located between the main beam and the feeding point; (4) The mitered corner' feed of figure 4 may also have abetted the formation of sidelobes due to diffraction about the mitered wall. The corner was designed from data for the intersection of two straight, closed waveguides; the waveguide here was neither straight nor closed.

The error in beamwidth of $3.6^{\circ}$ at the design frequency may also be caused by the fact that the correction term in (39) was neglected. This term would tend to decrease the wire spacing $p$ significantly in the region from $165^{\circ}$ to $215^{\circ}$, which would decrease energy leakage and in turn the beamwidth.

\section{Conclusions}

It has been shown that the flush-mounted leaky-wave antenna offers an excellent means to synthesize a Chebyshev pattern in the equatorial plane surrounding a finite metallic cylinder. Experimental results bear out the theory except for the problem of spurious large sidelobes.

Discussion of the experimental results suggests two immediate actions as a cure for this problem: (1) Inclusion of the correction term in (39), (2) use of an alternate feeding method such as a probe within the waveguide a quarter wavelength from the closed end.

Since the purpose of this report was to synthesize, as well as determine the requirements for synthesis of a specified radiation pattern under certain boundary conditions, investigation beyond these goals was not undertaken. Therefore, the other characteristics of the resulting antenna such as its impedance, efficiency, and gain have been left unevaluated.

Finally, although the azimuthal radiation pattern was the prime interest in this report, it is suggested that investigation of synthesis of an elevation pattern might also constitute an interesting research topic.

\section{Appendix A. Tabulation of Numerical Calculations}

The following is the numerical solution carried out for the design of the experimental antenna:

Cylinder radius, $a=7.0812$ in. (6 6 )

Height of aperture, $2 l=0.40 \mathrm{in}$. 


\begin{tabular}{|c|c|c|c|c|c|c|}
\hline$\phi(0)$ & $\alpha$ (NEPERS/ IN.) & $-\sigma$ (NEPERS / IN.) & $T(R A D /$ IN.) & $c^{\prime}$ & $W(\mid N)$. & $P(1 N)$ \\
\hline 005 & $7.67994(-07)$ & $8.84393(-07)$ & 3.49062 & $3.91241(03)$ & 0.8998 & 0.0313 \\
\hline 010 & $1.09548(-05)$ & $1.26151(-05)$ & 3.49062 & $1.03512(03)$ & 0.8990 & 0.0384 \\
\hline 015 & $4.56503(-05)$ & $5.25691(-05)$ & 3.49062 & $5.07040(02)$ & 0.8980 & 0.0454 \\
\hline 020 & $1.06075(-04)$ & $1.25607(-04)$ & 3.49062 & $3.28237(02)$ & 0.8970 & 0.0515 \\
\hline 025 & $1.83364(-04)$ & $2.11155(-04)$ & 3.49062 & $2.53137(02)$ & 0.8961 & 0.0562 \\
\hline 030 & $2.35431(-04)$ & $2.71113(-04)$ & 3.49062 & $2.23362(02)$ & 0.8955 & 0.0338 \\
\hline 035 & $2.38153(-04)$ & $2.74247(-04)$ & 3.49062 & $2 \cdot 22090(02)$ & 0.8955 & 0.0586 \\
\hline 040 & $1.89652(-04)$ & $2.18396(-04)$ & 3.49062 & $2.48903(02)$ & 0.8960 & 0.0566 \\
\hline 045 & $1.15057(-04)$ & $1.32495(-04)$ & 3.49062 & $3.19527(02)$ & 0.8969 & 0.0520 \\
\hline 050 & $4.88415(-05)$ & $5.62443(-05)$ & 3.49062 & $4.90393(02)$ & 0.8980 & 0.0458 \\
\hline 055 & $1.17362(-05)$ & $1.35150(-05)$ & 3.49062 & $1.00098(03)$ & 0.8990 & 0.0386 \\
\hline 060 & $7.85692(-07)$ & $9.04770(-07)$ & 3.49062 & $3.87551(03)$ & 0.8998 & 0.0314 \\
\hline 065 & $1.06820(-12)$ & 0.00000 & 3.49062 & $\infty$ & 0.9000 & 0.0250 \\
\hline 070 & $1.04908(-06)$ & $1.20805(-06)$ & 3.49062 & $3.36607(03)$ & 0.8997 & 0.0319 \\
\hline 075 & $1.40925(-05)$ & $1.62284(-05)$ & 3.49062 & $9.12853(02)$ & 0.8989 & 0.0394 \\
\hline 080 & $5.70402(-05)$ & $6.56853(-05)$ & 3.49062 & $4.53965(02)$ & 0.8978 & 0.0468 \\
\hline 085 & $1.31583(-04)$ & $1.51526(-04)$ & 3.49062 & $2 \cdot 98774(02)$ & 0.8967 & 0.0532 \\
\hline ०९० & $2.09090(-04)$ & $2.40779(-04)$ & 3.49062 & $2.37010(02)$ & 0.8958 & 0.0575 \\
\hline 095 & $2.44198(-04)$ & $2.81209(-04)$ & 3.49062 & $2.19352(02)$ & 0.8954 & 0.0592 \\
\hline 100 & $2.10031(-04)$ & $2.41864(-04)$ & 3.49062 & $2.36531(02)$ & 0.8958 & 0.0575 \\
\hline 105 & $1 \cdot 25266(-04)$ & $1.44252(-04)$ & 3.49062 & $3.06262(02)$ & 0.8967 & 0.0527 \\
\hline 110 & $4.32409(-05)$ & $4.97945(-05)$ & 3.49062 & $5.21291 \cdot(02)$ & 0.8981 & 0.0451 \\
\hline 115 & $4.71458(-05)$ & $5.42912(-06)$ & 3.49062 & $1.58027(03)$ & 0.8994 & 0.0355 \\
\hline 120 & $9.07968(-11)$ & 0.0000 & 3.49062 & $\infty$ & 0.9000 & 0.0250 \\
\hline 125 & $5.41350(-06)$ & $6.23398(-06)$ & 3.49062 & $1.47234(03)$ & 0.8993 & 0.0359 \\
\hline 130 & $1.25218(-04)$ & $1.44196(-04)$ & 3.49062 & $3.06262(02)$ & 0.8967 & 0.0527 \\
\hline 135 & $7.90178(-04)$ & $9.09939(-04)$ & 3.49062 & $1.21876(02)$ & 0.8918 & 0.0747 \\
\hline 140 & $2 \cdot 88687(-03)$ & $3.32441(-03)$ & 3.49063 & $6.36283(01)$ & 0.8843 & 0.1014 \\
\hline 145 & $7.69009(-03)$ & $8.85556(-03)$ & 3.49064 & $3.88000(01)$ & 0.8744 & 0.1320 \\
\hline 150 & $1.65789(-02)$ & $1.90911(-02)$ & 3.49072 & $2.61997(01)$ & 0.9626 & 0.1656 \\
\hline 155 & $3.06655(-02)$ & $3.53100(-02)$ & 3.49094 & $1.90115(01)$ & 0.8490 & 0.2014 \\
\hline 160 & $5.05201(-05)$ & $5.81628(-05)$ & 3.49147 & $1.45472(01)$ & 0.8352 & 0.2387 \\
\hline 165 & $7.60795(-02)$ & $8.75619(-02)$ & 3.49255 & $1.15918(01)$ & 0.8209 & 0.2770 \\
\hline 170 & $1.06681(-01)$ & $1.22716(-01)$ & 3.49441 & $9.53973(00)$ & 0.8068 & 0.3156 \\
\hline 175 & $1.41071(-01)$ & $1.62144(-01)$ & 3.49723 & $8.06800(00)$ & 0.7933 & 0.3539 \\
\hline 180 & $1.77229(-01)$ & $2.03483(-01)$ & 3.50104 & $6.99853(00)$ & 0.7809 & 0.3905 \\
\hline 185 & $2.11871(-01)$ & $2.42948(-01)$ & 3.50548 & $6.23747(00)$ & 0.7701 & 0.4234 \\
\hline 190 & $2.39572(-01)$ & $2.74392(-01)$ & 3.50958 & $5.74986(00)$ & 0.7622 & 0.4485 \\
\hline 195 & $2.51981(-01)$ & $2.88440(-01)$ & 3.51157 & $5.55760(00)$ & 0.7588 & 0.4594 \\
\hline 200 & $2 \cdot 38981(-01)$ & $2.73723(-01)$ & 3.50949 & $5.75936(00)$ & 0.7624 & 0.4480 \\
\hline 205 & $1.95285(-01)$ & $2.24072(-01)$ & 3.50326 & $6.57705(00)$ & 0.7751 & 0.4079 \\
\hline 210 & $1.30713(-01)$ & $1.50279(-01)$ & 3.49630 & $8.45117(00)$ & 0.7971 & 0.3428 \\
\hline 215 & $6.87288(-02)$ & $7.91098(-02)$ & 3.49220 & $1.22738(01)$ & 0.8247 & 0.2667 \\
\hline 220 & $2.73303(-02)$ & $3.14702(-02)$ & 3.49087 & $2.02004(01)$ & 0.8521 & 0.1939 \\
\hline 225 & $7.62880(-03)$ & $8.78498(-03)$ & 3.49064 & $3.89577(01)$ & 0.8746 & 0.1317 \\
\hline 230 & $1.21423(-03)$ & $1.39826(-03)$ & 3.49062 & $9.82722(01)$ & 0.8898 & 0.0822 \\
\hline 235 & $5.24186(-05)$ & $7.04784(-05)$ & 3.49062 & $4.73114(02)$ & 0.8979 & 0.0463 \\
\hline 240 & $8.80574(-10)$ & 0.0000 & 3.49062 & $\infty$ & 0.9000 & 0.0250 \\
\hline 245 & $4.57349(-05)$ & $5.23377(-05)$ & 3.49062 & $5.06776(02)$ & 0.8980 & 0.0454 \\
\hline 250 & $4.19570(-04)$ & $4.80160(-04)$ & 3.49062 & $1.67313(02)$ & 0.8940 & 0.0656 \\
\hline 255 & $1.21662(-03)$ & $1.40101(-02)$ & 3.49062 & $9.81775(01)$ & 0.8898 & 0.0823 \\
\hline 260 & $2.04403(-03)$ & $2.35382(-03)$ & 3.49063 & $7.56770(01)$ & 0.8868 & 0.0930 \\
\hline 265 & $2.38331(-03)$ & $2.74453(-03)$ & 3.49063 & $7.00617(01)$ & 0.8858 & 0.0966 \\
\hline 270 & $2.04653(-03)$ & $2.34671(-03)$ & 3.49063 & $7.56347(01)$ & 0.8868 & 0.0931 \\
\hline 275 & $1.29071(-03)$ & $1.48633(-03)$ & 3.49062 & $9.53074(01)$ & 0.8895 & 0.0834 \\
\hline 280 & $5.60181(-04)$ & $6.45080(-04)$ & 3.49062 & $1.44768(02)$ & 0.8931 & 0.0694 \\
\hline 285 & $1.38462(-04)$ & $1.59447(-04)$ & 3.49062 & $2.91332(02)$ & 0.8966 & 0.0536 \\
\hline 290 & $1.03083(-05)$ & $1.18707(-05)$ & 9062 & $1.06751(03)$ & 0.8991 & 0.0381 \\
\hline 295 & $1.05081(-11)$ & 0.0000 & 3.49062 & $\infty$ & 0.9000 & 0.0250 \\
\hline 300 & & $8.89010(-06)$ & 3.49062 & $1.23301(03)$ & 0.8992 & 0.0371 \\
\hline 305 & $1.15331(-04)$ & $1.32811(-04)$ & 3.49062 & $3.19154(02)$ & 0.8969 & 0.0520 \\
\hline 310 & $4.80176(-04)$ & $5.52956(-04)$ & 3.49062 & $1.56371(02)$ & 0.8936 & 0.0673 \\
\hline 315 & $1.13250(-03)$ & $1.30415(-03)$ & 3.49062 & $1.01768(02)$ & 0.8902 & 0.0809 \\
\hline 320 & $1.87084(-03)$ & $2 \cdot 15438(-03)$ & 3.49062 & $7.91166(01)$ & 0.8874 & 0.0910 \\
\hline 325 & $2.35653(-03)$ & $2.71369(-03)$ & 3.49063 & $7.04631(01)$ & 0.8859 & 0.0964 \\
\hline 330 & $2.33762(-03)$ & $2.69191(-03)$ & 3.49063 & $7.07457(01)$ & 0.8859 & 0.0962 \\
\hline 335 & $1.82625(-03)$ & $2.10304(-03)$ & 3.49063 & $8.00825(01)$ & 0.8876 & 0.0905 \\
\hline 340 & $1.08875(-03)$ & $1.25376(-03)$ & 3.49062 & $1.03788(02)$ & 0.8904 & 0.0802 \\
\hline 345 & $4.56217(-04)$ & $5.25361(-04)$ & 3.49062 & $1.60452(02)$ & 0.8938 & 0.0667 \\
\hline 350 & $1.09533(-04)$ & $1.26134(-04)$ & 3.49062 & $3.27580(02)$ & 0.8970 & 0.0516 \\
\hline 355 & $7.68006(-06)$ & $8.84406(-06)$ & 3.49062 & $1 \cdot 23674(03)$ & 0.8992 & 0.0371 \\
\hline 360 & $1.38509(-16)$ & 0.0000 & 3.49062 & $\infty$ & 0.9000 & 0.0250 \\
\hline
\end{tabular}


Wire diameter, $d / 2=0.01264 \mathrm{in}$.

Design frequency, $f=10.0 \mathrm{Gc} / \mathrm{s}$

Design wavelength, $\lambda=1.1802 \mathrm{in}$.

Free space propagation constant, $k=5.32384 \mathrm{rad}$./in.

Design guide wavelength, $\lambda_{g}=1.5631 \mathrm{in}$.

Guide propagation factor, $\beta=4.01967 \mathrm{rad}$./in.

\section{References}

Borgnis, F. E., and C. H. Papas, Electromagnetic waveguides, Encyclopedia of Physics XVI, 364-369 (Springer-Verlag, Berlin, 1958).

Duhamel, R. H., Optimum patterns for endfire arrays, Proc. IRE 41, No. 5, 6j3-659 (May 1953).

Gladstone, L. O., and A. A. Oliner, Leaky wave antennas I: Rectangular waveguides, IRE Trans. Ant. Prop. AP-7, 307-319 (Oct. 1959).

Honey, R. C., A flush-mounted leaky-wave antenna with predictable patterns, IRE Trans. Ant. Prop. AP-y, 320-328 (Oct. 1959).

Silver, S., Microwave antenna theory and design, 282-283 (McGraw-Hill Book Co. Inc., New York, 1949).

Silver, S., and W. K. Saunders, The external field produced by a slot on an infinite circular cylinder, J. Appl. Phys. 21, 153-158 (Feb. 1950).

Sommerfeld, A., Partial differential equations in physics (Academic Press, Inc., New York, 1949).

Wait, J. R., Electromagnetic radiation from cylindrical structures (Pergamon Press, Ltd., London, 1959).

Wait, J. R., and J. Householder, Pattern synthesis for slotted-cylinder antennas, J. Research NBS 63D (Radio Prop.) No. 3, 303-313 (Nov.-Dec. 1959).

(Paper 66D6-236) 